\title{
Laying the digital and analytical foundations for Canada's future health care system
}

\author{
Muhammad Mamdani PharmD MPH, Andreas Laupacis MD MSc
}

- Cite as: CMAJ 2018 January 8;190:E1-2. doi: 10.1503/cmaj.170955

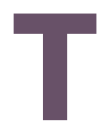

he growing incorporation of advanced analytics (the use of computationally intensive data analysis approaches) into an increasingly digital health care environment in Canada is promising, but vision, planning and further investment of financial and human resources and social capital are required to realize their full potential. Evidence to support the contention that better patient outcomes and efficiency are achieved through advanced analytics remains sparse. For many, the investments required may seem too great and the benefits too uncertain to justify them. However, advanced analytics have great potential to inform patient prognosis, improve treatment and patient outcomes, and enhance the efficiency of our health care system.

Adoption of electronic medical records by family physicians more than tripled between 2005 and 2015, from 24\% to $77 \%$, and $70 \%$ of family physicians report improved quality of care as a result. ${ }^{1}$ National and provincial initiatives such as Canada Health Infoway, Alberta Netcare and ConnectingOntario have made considerable strides in centralizing large amounts of digital data for clinical management. For example, about 89000 clinicians access ConnectingOntario electronic health records, which provide realtime access to patient health information from participating outpatient clinics and hospitals. ${ }^{2}$

Recent advances in information technology with respect to storage, management and data processing have renewed interest in applying advanced analytics to large clinical data repositories. Concurrently, the sophistication of clinical decision support tools at the point of care has evolved from relatively simple alerts and reminders promoting optimal clinical practice to complex machine learning algorithms that adapt to patient conditions in real time; for example, to predict admissions to the intensive care unit among patients in hospital. ${ }^{3}$ Several innovations offer insight into the potential gains from greater investment in digital health care and analytics.

Diagnostic innovations include emerging statistical learning approaches capable of rapidly detecting diabetic retinopathy and macular edema, ${ }^{4}$ as well as certain skin cancers, ${ }^{5}$ through image pattern recognition as accurately as - and, in some cases, better than - expert clinicians. Enlitic, a machine learning company, has recently partnered with a major radiology service provider to bring deep learning diagnostics to the Australian and Asian markets to assist radiologists in diagnosis, ${ }^{6}$ which may

\section{KEY POINTS}

- Although many sectors have rapidly embraced recent advances in data management and advanced analytics in transformative ways, our health care system is struggling to keep pace.

- The health care sector is among the most data rich, yet only a handful of health care entities are making investments in data analytics to glean benefits, such as analytical algorithms that diagnose disease faster and better than expert clinicians.

- Critical factors for success include data availability, underlying architecture, quality assurance and governance, as well as issues related to privacy and security, capacity and skills in managing and analyzing health care data, and a "data-ready" culture.

- There is a sense of urgency for the Canadian health care system to consider more thoughtfully how it can best prepare for a radically different future in which technology will play an increasingly fundamental role in the lives of the health care community and the patients we serve.

greatly improve access to standardized, accurate and efficient diagnostic services.

Advanced analytical approaches have also been used to increase precision in predicting the likelihood of death or disability at hospital discharge among patients with stroke. An observational study showed that precision was about $90 \%$ using a sophisticated risk prediction algorithm, compared with about $17 \%$, based on clinical judgment. ${ }^{7}$

Computational platforms have great potential to transform and streamline patient management, through sophisticated analysis of patient-level data such as radiologic images, laboratory values, genomics data and pharmacotherapy, as well as text notes, images and evidence from clinical research. For example, the Veterans Health Administration in the United States implemented individualized clinical risk prediction scores in real time to guide clinical management decisions among patients with congestive heart failure, which led to a $27 \%$ reduction in emergency department visits. ${ }^{8}$

Leading health care organizations such as Intermountain Healthcare and Kaiser Permanente in the US have a long-standing history of incorporating advanced analytics, including risk prediction and diagnostic tools, into their information systems to assist clinical management to improve clinical performance. These 
organizations have made considerable investments in data infrastructure and in skilled data scientists in their quest to provide high-quality, efficient health care.

Are Canadian health care providers in a position to realize the benefits of advanced analytics? A recent Canadian Institute for Health Information report outlined key building blocks in leveraging health care data to improve health, including data availability, underlying architecture, quality assurance and governance, as well as issues related to privacy and security, capacity and skills in managing and analyzing health care data, and a "data-ready" culture enabling data analytics. ${ }^{9}$

Building the required infrastructure is not without its challenges. Advanced analytics can be useful only if they are based on good data. Reliable data that can support valid data-driven insights into clinical practice depend on good data infrastructure and strong security and governance processes, ${ }^{10}$ in addition to well-trained data scientists, all of which require investment. Recruitment of large numbers of data scientists in a highdemand market may impose substantial costs for the public sector. Investments must be justified. Despite the immense potential for advanced analytics to transform health care delivery, to our knowledge a clear return on investment has yet to be shown. However, without these investments, we may never be able to explore their potential.

Strong leadership is required to bridge the gap between data scientists and end users. Effective multidisciplinary teams that integrate skilled data scientists with team members such as administrative professionals, information technology experts, implementation experts, clinicians and patients are critical to conducting advanced analytics that are useful for management and clinical practice.

Furthermore, we need a "data-friendly" culture, with dataliterate senior management and clinicians who are receptive to, and not threatened by, assistance from advanced analytics in their work. However, as with any intervention, evaluation and monitoring of outcomes associated with the incorporation of advanced analytics into clinical practice are essential. Unexpected adverse outcomes may occur. For example, a recent cluster randomized trial examining the impact of a clinical prediction rule in assisting with the decision of whether to admit patients with community-acquired febrile urinary tract infection reported a fourfold increase in secondary hospital admission rates after initial outpatient treatment in the intervention arm relative to the control arm. ${ }^{11}$ Numerous factors, including the approach to derivation and validation of the clinical prediction rule, as well as compliance with the rule's recommendations, may have contributed to unexpected adverse outcomes in this study. Understanding the strengths and limitations of available data and resulting analyses, in addition to employing effective communication strategies, will be critical to driving and supporting positive change and to realizing the promises of advanced analytics in Canadian health care.

As other industries are rapidly developing robust digital environments and gleaning transformative benefits from advanced analytics, ${ }^{12}$ health care is being left behind. It should be stressed that some initiatives intended to improve patient care using advanced analytics will certainly fail. However, the potential benefits are so great that Canadian health care organizations must make the substantial investments needed to implement and learn from advanced analytics. Failures should be viewed as learning opportunities and the insights gained should be used to strengthen future endeavours. We need champions with a bold vision to lay the digital and analytical foundations for our future health care system.

\section{References}

1. Report on digital health: Canadians embracing digital health. Toronto: Canada Health Infoway; 2015. Available: https://www.infoway-inforoute.ca/en/ component/edocman/2815-infographic-report-on-digital-health-canadians -embracing-digital-health/view-document? Itemid=0 (accessed 2017 Aug. 10).

2. ConnectingOntario. Toronto: eHealth Ontario; 2017. Available: http://www ehealthontario.on.ca/en/for-healthcare-professionals/connectingontario (accessed 2017 Aug. 10).

3. Yoon J, Alaa A, Hu S, et al. ForecastICU: a prognostic decision support system for timely prediction of intensive care unit admission. Proceedings of [J Mach Learn Res W\&CP] the 33rd International Conference on Machine Learning; 2016 June 19-24; New York. Available: http://proceedings.mlr.press/v48/yoon16.pdf (accessed 2017 Aug. 10).

4. Gulshan V, Peng L, Coram M, et al. Development and validation of a deep learning algorithm for detection of diabetic retinopathy in retinal fundus photographs. JAMA 2016;316:2402-10.

5. Esteva A, Kuprel B, Novoa RA, et al. Dermatologist-level classification of skin cancer with deep neural networks. Nature 2017;542:115-8.

6. Enlitic and Capitol Health Announce Global Partnership: leveraging deep learning to enhance physician care for millions of patients [press release]. San Francisco: Enlitic; 2015. Available: http://www.enlitic.com/press-release-10272015.html (accessed 2017 Dec. 15).

7. Saposnik G, Cote R, Mamdani M, et al. JURaSSiC: accuracy of clinician vs risk score prediction of ischemic stroke outcomes. Neurology 2013;81:448-55.

8. Parikh RB, Kakad M, Bates DW. Integrating predictive analytics into high-value care: the dawn of precision delivery. JAMA 2016;315:651-2.

9. Better information for improved health: a vision for health system use of data in Canada. Ottawa: Canadian Institute for Health Information and Canada Health Infoway; 2013. Available: https://www.cihi.ca/en/hsu_vision_report_en.pdf (accessed 2017 Aug. 10).

10. Egelstaff R, Wells M. Data governance frameworks and change management. Stud Health Technol Inform 2013;193:108-19.

11. Stalenhoef JE, van der Starre WE, Vollaard AM, et al. Hospitalization for community-acquired febrile urinary tract infection: validation and impact assessment of a clinical prediction rule. BMC Infect Dis 2017;17:400.

12. Greenwald T. How Al Is transforming the workplace. Wall Street Journal [New York] 2017 Mar. 10. Available: www.wsj.com/articles/how-ai-is-transforming-the -workplace-1489371060 (accessed 2017 Dec. 18).

Competing interests: Andreas Laupacis holds a Canada Research Chair in Health Policy and Citizen Engagement. No other competing interests were declared.

This article has been peer reviewed.

Affiliations: Editorial Advisory Board member (Mamdani) CMAJ; Li Ka Shing Knowledge Institute (Mamdani, Laupacis), St. Michael's Hospital; Faculty of Medicine (Mamdani, Laupacis), University of Toronto; Institute of Health Policy, Management, and Evaluation (Mamdani, Laupacis), University of Toronto; Institute for Clinical Evaluative Sciences (Mamdani, Laupacis); Leslie Dan Faculty of Pharmacy (Mamdani), University of Toronto, Toronto, Ont.

Disclaimer: Muhammad Mamdani is a member of the CMAJ editorial advisory board and was not involved in the editorial decision-making process for this article.

Correspondence to: Muhammad Mamdani, mamdanim@smh.ca 